\title{
QoS-Aware LTE Downlink Scheduler for VoIP with Power Saving
}

\author{
M. Sajid Mushtaq \\ Image, Signal and Intelligent Systems Laboratory-LISSI \\ Dept. of Networks and Telecoms, IUT C/V \\ University of Paris-Est Creteil (UPEC), France
}

\author{
Abdussalam Shahid and Scott Fowler \\ Mobile Telecommunications \\ Dept. of Science and Tech. \\ Linköping University, Norrköping, Sweden
}

\begin{abstract}
The addition of emerging multimedia services such as Skype, GTalk, multi-user interactive gaming in cellular communication systems has created new challenges for resource allocation and power optimization. The requirement of efficient resource allocation is more important in downlink than uplink due to high traffic flows. These multimedia applications require more power; therefore power optimization has gained a key role in future communication systems. In this paper, a downlink scheduling technique in Long Term Evolution (LTE) is proposed that improves the Quality of Service (QoS) for multimedia services in relation with use of the power saving scheme at the User Equipment (UE) i.e. Discontinuous Reception (DRX). The DRX effectively improves power consumption at the cost of QoS degradation due to higher packet delays and packet losses. The proposed scheme considers key QoS parameters during scheduling with fair resource allocation while minimizing packet delay and packet loss even in a power saving environment.
\end{abstract}

Keywords: QoS; LTE; Power Saving; Scheduling; VoIP; Downlink; Light Sleep; DRX; Multimedia.

\section{INTRODUCTION}

Recent advance research has developed a large variety of smart mobile devices, which are powerful enough to support a wide range of multimedia traffic (e.g. VoIP, video streaming, multiplayer interactive gaming) and also legacy mobile services (e.g. voice, SMS, MMS). These new multimedia applications require high data rates and power to provide better Quality of Service (QoS). However, due to the low transmission rate and high service costs, the $3 \mathrm{G}$ (third generation) technology has been unsuccessful in delivering ubiquitous/high-speed mobile broadband.

To address the mobile broadband requirements, the 3GPP introduced the new radio access technology Long Term Evolution (LTE) which has the capability to move towards fourth generation (4G) wireless systems. LTE is designed to be a high data rate and low latency system that aiming to support different types of services, including web browsing, FTP, HD video streaming, VoIP, online multi-user interactive gaming and real time video. However, the use of enriched $4 \mathrm{G}$ services is still limited because the receivers of these services require computationally complex circuitry that drains the user equipments (UE) battery power quickly.

Optimization of power consumption at the UE is one of the main concerns in wireless networks. The idea is to improve UEs battery life so that the UE does not run out of power too quickly. To save power at the UE, LTE specification includes the concept of Discontinuous Reception (DRX) with Light Sleep and Deep Sleep modes [5]. DRX Light Sleep mechanism puts the UEs receiver into sleep mode for shorter durations. DRX Light Sleep does not shut down the UEs receiver completely; rather it consumes power less than the active mode of operation. The receiver switches between active and sleep mode periodically to receive scheduled packets. If the UE does not receive packets for a long period of time, then the receiver is turned off completely and the UE goes into DRX Deep Sleep mode. In DRX Deep Sleep mode, the receiver does not require any power and sleep durations become longer than DRX Light Sleep. The DRX Sleep mode has a direct influence on the QoS for multimedia traffic because more power saved will result in more packet delays or packet loss. To address this problem, there is a need to optimize the DRX parameters, to maximize power saving without incurring network re-entry and packet delay. In particular, care should be exercised for real-time services.

In this paper, we proposed a downlink scheduling scheme for delay sensitive traffic (VoIP). The proposed scheme uses opportunistic scheduling approach that calculates the priorities of UEs and assigns resources based on following features: channel condition, average throughput, UE buffer status, GBR/non-GBR traffic, UE DRX status and packets delay at the eNodeB buffer. The proposed scheme is evaluated in normal operation mode and power saving mode and the impact of power saving on QoS.

\section{RELATED WORK}

There are many QoS factors that directly affect the performance of network and UE. The scheduling and power saving are two main factors that have gained greater importance after the introduction of newly emerging multimedia applications because these applications require more radio resources and consume high power for providing better QoS.

\section{A. Scheduling Schemes}

Scheduling is a process of allocating the physical resources among UEs and it guarantees QoS of multimedia service. The aim of a scheduling scheme is to maximize the overall system throughput while keeping fairness, delay and packet loss rate within QoS requirements. The scheduling decisions 
can be made on the basis of QoS parameters, Channel Quality Indicator (CQI), resource allocation history, buffer status both at the eNodeB and UE. The Best CQI (B-CQI) algorithm chooses the UEs that report the highest downlink Signal to Noise Ratio (SNR) values to corresponding eNodeB; thus, utilizes the radio resources efficiently among the UEs with good channel condition. On the other hand, the UEs experiencing bad channel conditions would never get resources [9]. As a result, overall system throughput increases but it outcomes in starvation of resources for some UEs specially the UE far away from the eNodeB. Thus, the B-CQI algorithm performs good in terms of throughput but poor in terms of fairness among the UEs. In order to cope with the fairness problem in BCQI algorithm, the Round Robin (RR) algorithm was designed based on queues. It allocates the equal packet transmission time to each UE to meet the fairness [9]. As a consequence, the whole system throughput degrades because this algorithm does not consider channel conditions. To balance out the constraints of high throughput and fairness, the Proportional Fair (PF) algorithm was proposed. The scheduling strategies which are based on PF algorithm focus on tradeoff between maximum average throughput and fairness.

In [7],the packet scheduling algorithm proposed, which investigate QoS for real time services by 1 ) CQI feedback on uplink, 2) it assumes that every UE has limited buffer length and buffer status is also sent on the uplink that contributes in making the scheduling decisions, 3) treats real time and nonreal time UEs traffic separately. This algorithm does not take into account packet delay as a result packet loss ratio may increase. In [1] the time and frequency domain scheduling is proposed, which is implemented in two layers to keep the fairness of radio resource and improve the throughput. In LTE, Guaranteed Bit Rate (GBR) and delay constraints are the key attributes that determine QoS but this scheme does not consider these factors. Despite these QoS methods for LTE, they do not take into account power saving mechanism, which is an important entity of LTE networks.

\section{B. Power Saving mode (DRX Mechanism)}

The DRX is an important mechanism that increases the UE battery life by observing the UE activity. When there is no data transmission, the DRX saves UEs power by turning off the wireless transceiver. In the DRX mechanism, packet delays increase significantly as a UE switches to sleep mode for a period. More information about how DRX mechanism works can be found in [10].

In [2] the DRX-aware scheduling is proposed, which includes DRX status as scheduling decision parameter to reduce packet delay caused by DRX sleep duration. The scheduling priority is directly proportional to delay of a head of line packet delay in relation to the remaining active time before a UE enters into sleep mode. In [3] semi-persistent scheduling scheme for VoIP is developed using DRX. First, it organizes the UEs into scheduling candidate set (SCS) based on UE buffer information at the eNodeB, DRX status and persistent resource allocation pattern. It calculates the priority metric for the UEs in SCS by favoring the UEs who require retransmission then the UEs whose packet delay of unsent packet at the eNodeB buffer is close to delay threshold.

Both schemes presented in [2], [3] use DRX mechanism to optimize power usage and offer solutions to the problems caused by the sleep interval of increased packet delay and packet loss. However, both schedulers do not consider GBR requirement of UEs.

\section{Proposed SCHEME}

There is a tradeoff between the QoS and power saving. To balance between QoS and power consumption, a new scheduling technique is proposed to ensure QoS of Real Time (RT) and Non-Real Time (NRT) traffic services while efficiently utilize the power. Most of scheduling algorithms discussed in Section II, achieve multiuser diversity that uses an opportunistic approach to assign resources to UEs by considering channel conditions. The high system throughputs can be achieved by assigning resources only to those UEs who have good channel condition; however, these techniques fail to fulfill fairness and the UEs QoS requirements. To deal with these problems, other parameters are required in order to balance between spectral efficiency and UE requirements.

The proposed scheduler uses opportunistic scheduling approach that calculates the priorities of UEs and assigns resources to them. This opportunistic scheduling approach is based on the six important scheduling dependencies that have the greater impact on QoS and Power saving mechanism :

1) Channel condition: Scheduler estimates data rates and modulation scheme for each UE on every sub-band. Estimation is based on CQI reports sent by the UEs in the uplink, which include information about downlink SINR.

2) Average throughput: The averaged data rate experienced by each UE for a time window. By keeping track of the UE throughput history the scheduler will be able to give more resources to those UE which were lacking in the past to fulfill their requirements and as a result fairness among the UEs would also increase.

3) UE buffer status: Every UE has a finite buffer length to store received packets. Packet losses can occur due to insufficient space in a buffer. In the proposed algorithm, buffer length at the UE is assumed to be limited and the scheduler gives high priority to the UEs, who have more buffer space to avoid packet loss. Similarly, the UEs who have the smaller spare buffer would get low priority to minimize packet loss.

4) GBR/non-GBR: Schedulers require treating RT and NRT services separately. GBR is an important parameter for RT serviced UEs. If a UE experiences data rate lower than defined by the GBR, the scheduler must allocate more resources to that UE.

5) DRX status: DRX is an effective power saving technique to prolong UE battery life. There is a tradeoff between power conservation and QoS; more power savings result 
in higher transmission delays and packet losses. To address this issue, the proposed algorithm considers DRX status to bind the delays within thresholds according to QCI characteristics of LTE.

6) Delay: It is required by the scheduler to increase the priority of the UE which has the oldest packet delay in the eNodeB buffer is closed to delay threshold so that packet loss can be minimized.

\section{A. Scheduler Architecture}

The main entities involved in a downlink scheduling algorithm are shown in Figure 1. In Figure 1, eNodeB is shown the left side with Layer 1 - Layer 3 and UEs shown at the right side. The information flows shown in the Figure 1 with solid lines are used both by the traditional and proposed scheduling algorithms. While information flows shown with dash lines are used only by the proposed scheduling algorithm.

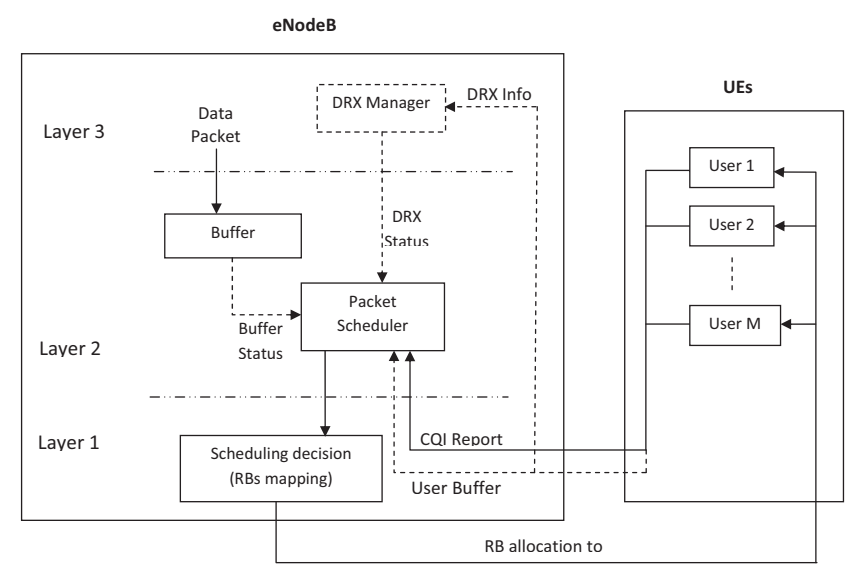

Figure 1: Entities involved in downlink packet scheduler.

The proposed scheduler at Layer 2 acquires CQI reports from the UEs in order to estimate the channel conditions while UEs buffer status are also received to avoid packet loss because the receiver buffer at the UE is assumed to be limited. A set of buffers at the eNodeB stores the packets for each UE to be scheduled. The proposed scheduler attempts to minimize packet losses by prioritizing the UEs who has the oldest packet in the eNodeB buffer. Each UE sends its DRX information to the DRX manager, and the DRX manager sends the DRX status to the packet scheduler who determines the remaining active and Sleep mode time for each UE. By considering six scheduling dependencies, the proposed scheduler assigns resources to the UEs through Physical Downlink Control Channel (PDCCH). This allows the proposed scheduling algorithm to keep the packet within delay bounds and effectively minimize packet delays and packet loss rate.

\section{B. Scheduling Algorithm}

The proposed algorithm selects and assigns available Resource Blocks (RBs) to UEs according to the priority ma- trix. The priority matrix is calculated by considering the six scheduling dependencies which were previously described. The priority values for each RB are estimated for every UE; scheduler assigns RB to a UE whose priority value is highest among all other UEs for that specific RB.

To calculate the priorities, the algorithm first estimates maximum achievable throughputs for every $\mathrm{RB}$ if assigned to UEs according to channel conditions reported by UEs. In order to balance between system throughput and fair resource distribution the proposed scheduler utilizes the property of Proportional Fair (PF) which is defined in [9].

$$
\begin{gathered}
\text { fair_factor }_{i}=\frac{\text { achievable_throughput }_{i j}}{\text { average_throughput }_{i}} \\
R_{i}(t)=\left(1-\frac{1}{t_{c}}\right) * R_{i}(t-1)+\frac{1}{t_{c}} * r_{i}(t-1)
\end{gathered}
$$

In (1) achievable_throughput $t_{i j}$ represents theoretical achievable throughput of $R B_{j}$ if assigned to $U E_{i}$ at Transmission Time Interval (TTI). In (2) $R_{i}$ represents the average_throughput $t_{i}$ of $U E_{i}$ over a window $t_{c}$ at every TTI and $r_{i}$ is achievable throughput of $U E_{i}$.

In the proposed scheduling algorithm, the UE buffer status report (BSR) is considered to provide immunity to packet loss due to the buffer overflow. The buffer status reporting is achieved by sending UE buffer information on the uplink. This information includes the receiver buffer length $\mathrm{L}_{b u f f}$ and the length of used space at buffer $\mathrm{N}_{c u r r}$. When the UE buffer is empty then $\mathrm{N}_{\text {curr }}$ is initialized to 0 and increments in step of 1 at arrival of each packet at UE buffer. When the buffer fills up completely, $\mathrm{N}_{c u r r}$ reaches buffer length $\mathrm{L}_{\text {buff }}\left(\mathrm{N}_{c u r r}\right.$ $=\mathrm{L}_{b u f f}$ ) after that it will be flushed back to 0. According to [7], the BSR factor $b u f f i$ for $\mathrm{UE}_{i}$ at every TTI is calculated as

$$
b u f f_{i}=\frac{L_{b u f f_{i}}-N_{\text {curr }_{i}}}{L_{b u f f_{i}}}
$$

The priority function $\mathrm{P}_{i j}$ calculates priorities of Non-Real Time (NRT) and Real Time (RT) services from equations (4) and (5) respectively.

$$
\begin{aligned}
P_{i j} & =\delta_{i}\left(\text { fair }_{\text {factor }_{i}}+\text { buff }_{i}\right), \quad i \text { is NRT UE } \\
P_{i j} & =\text { delay_factor }_{i} \delta_{i}\left(\text { fair_factor }_{i}\left(\frac{\text { GBR }}{\text { average_throughput }_{i}}\right)^{\varnothing}\right. \\
& \left.+ \text { buff }_{i}\right), \quad i \text { is RT UE }
\end{aligned}
$$

where $\varnothing$ is a tunable exponential factor for GBR and $\delta$ is a DRX status indicator for each UE. The $P_{i j}$ is the priority matrix for each $R B_{j}$ if assigned to $U E_{i}$ while fair $_{-}$factor $_{i}$ and $b u f f_{i}$ are in accordant to (1) and (3) respectively. GBR is the guaranteed bit rate requirement for GBR UEs. The tunable exponential factor can be used to adjust the preference of GBR UEs; if a UE is achieving lower than average throughput required by the GBR, then the scheduler will increase priority of that UE to fulfill the GBR requirement and vice versa. 
The delay_factor ${ }_{i}$ is a priority multiplier that increases the priority of UEs whose data residing in the eNodeB buffer is close to delay thresholds; higher waiting time in the buffer means higher priority to prevent packet loss. The factors of delay_factor $_{i}$ and GBR are irrelevant for NRT traffic because NRT traffic is not delay sensitive, and they do not require minimum data rates to guarantee.

The proposed scheduling algorithm is designed in conjunction with DRX mechanism as to fully exploit high bandwidth efficiency of LTE. The DRX manager at eNodeB shares DRX status with the UEs. On each TTI, the scheduler must consider only the UEs who are in active mode of operation then allocate resources for data transmission, and this is achieved by including the DRX status in priority criteria. DRX status $\delta$ defines a state of UE, when a UE is the in-active mode $\delta=1$. When a UE is in Sleep mode $\delta=0$ makes that UE out of the scheduling competition. Thus, the scheduler helps reducing resource wastage by considering only the UEs who are in the active state.

\section{Simulation Setup}

In this paper a single hexagon cell with only one sector is considered, which has $5 \mathrm{MHz}$ system bandwidth and operating at $2 \mathrm{GHz}$ operating frequency. The eNodeB is considered to be static, which is serving 15 VoIP traffic UEs who are uniformly distributed within the sector and allowed to move randomly. These UEs can be considered as pedestrians moving with speed of $5 \mathrm{~km} / \mathrm{h}$. The VoIP traffic model is considered due to its fastest growing since last few years. Additionally, fading models are used to simulate realistic channel conditions. To save power, Light DRX Sleep mechanism is implemented on the UEs because finite buffer length is considered at eNodeB for each UE [4]. If Deep Sleep mode is used, then there are chances of buffer overflow at the eNodeB because the number of packets being created would be much higher than packets being scheduled. In this paper DRX On-duration and In-Active parameters are set to 1 TTI and 4 TTIs but they can vary as done in [10]. The power saving effect on QoS will be presented and discussed in a later section. Other important simulation parameters are listed in Table I.

\section{Simulation Results}

The performance of the proposed algorithm will be evaluated and compared with three traditional scheduling algorithms Proportional Fair (PF), Round Robin (RR) and Best CQI (BCQI) in both normal (no DRX) mode and power saving mode. The evaluation and comparison are done in same simulation environment and parameter.

Evaluation will be done on key performance evaluation parameters; System Throughput, Throughput Fairness Index, Packet Delay and Packet Loss Rate. Before presenting the results, it is better to define these performance evaluation parameters.

- System Throughput (Downlink): It is the accumulated data rates of all the UEs in bits/s. For this study work only downlink traffic is considered.
Table I: Main Simulation Parameters

\begin{tabular}{c|c}
\hline Parameters & Values \\
\hline eNodeB radius & $250 \mathrm{~m}$ \\
\hline Number of sectors per eNodeB & 3 \\
\hline Target area & Single sector \\
\hline Number of UEs & 15 \\
\hline eNodeB total TX power & $10 \mathrm{~W}$ \\
\hline Number of antennas (SISO) & Fast fading \\
\hline Fading models & $5 \mathrm{~km} / \mathrm{h}$ \\
\hline UE Speed & $2 \mathrm{GHz}$ \\
\hline Operating frequency band & $5 \mathrm{MHz}$ \\
\hline System channel bandwidth & 25 \\
\hline Number of RBs & 2 \\
\hline$\varnothing$ & $25 \mathrm{kbps}$ \\
\hline GBR & Every TTI \\
\hline CQI reporting & VoIP \\
\hline Traffic model & $20 \mathrm{~ms}$ \\
\hline VoIP packet generation interval & $100 \mathrm{~ms}$ \\
\hline VoIP delay threshold & DRX Sleep \\
\hline Power saving mechanism & $1 \mathrm{TTI}$ \\
\hline DRX on duration & $4 \mathrm{TTIs}$ \\
\hline DRX In-Active duration & $0,7,10,20,30,40,50(\mathrm{~ms})$ \\
\hline DRX Sleep duration &
\end{tabular}

- Throughput Fairness Index: Fairness can be defined in terms of resource allocation or throughput. Jains equation is used to obtain the throughput fairness index. In [6], fairness index $J$ for $n$ UEs is defined as

$$
J\left(x_{1}, x_{2}, \ldots, x_{n}\right)=\frac{\left(\sum_{i=1}^{n} x_{i}\right)^{2}}{n \sum_{i=1}^{n} x_{i}^{2}}
$$

where $x_{i}$ is the throughput for the $i^{t h}$ UE. The best case can give a maximum value of 1 which means all the UEs achieved exactly same throughput. When the difference between UEs throughput increase then the value of Jain's equation decreases.

- Packet Delay: It is the difference in time when a packet is created and when UE acknowledges that packet.

Packet Delay $=$ Created time - Acknowledged time

- Packet Loss Rate (PLR): It is the ratio of the number of packets lost to the number of packets sent. A packet is considered lost if the UE does not acknowledge it within the delay threshold. Delay threshold for VoIP is $100 \mathrm{~ms}$, and packet loss rate is $10^{-2}$ according to LTE QCI characteristic [8].

$$
\text { PLR }=\frac{\text { Number of packets lost }}{\text { Number of packets sent }}
$$

\section{A. Normal Operation Mode}

All the schedulers are used in the same simulation setup as presented in Table I. The receivers of all the UE are switched-on all the time that means no power is being saved by the UEs. The traditional schedulers which are designed to work in non-DRX environment are being compared with Proposed Scheme. However, the Proposed Scheme specially considers active and Sleep modes of UEs. Therefore, other schedulers may overwhelm the Proposed Scheme in one or more performance evaluation parameters. 


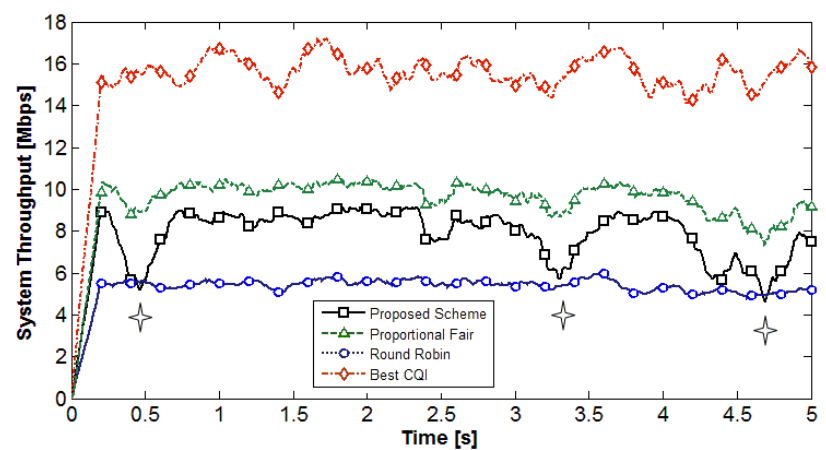

Figure 2: Downlink System Throughput with no DRX Sleep.

Figure 2, shows systems throughput performance when the simulation is run for 5000 TTI. The results show that Best CQI (B-CQI) scheduler performed the best because it chooses the UEs, which have the best channel conditions in the uplink through CQI feedbacks. The PF scheduler performed the second best in this regard because it tries to balance the system throughput with the fairness. The Proposed Scheme performed not as good as B-CQI and PF scheduler because it is not designed to maximize system throughput rather, it designed to provide good QoS. The three markers point to the time when the Proposed Schemes system throughput performance degraded significantly. The reason is the throughput of some UEs had started to go below the GBR limit due to bad channel condition, and the scheduler tried to compensate it by assigning more resources. The RR scheduler performed not so well, but its throughput is more stable than any other scheduler because it treats all the UEs equally regardless of their channel conditions or requirements.

Figure 3 shows throughput fairness indexes calculated through equation(6). The Proposed Scheme achieved significantly higher and stable fairness during simulation. The PF scheduler does not take into account GBR and delays constraints. Therefore, the PF scheduler is following the Proposed Scheme curve with lower fairness index. The RR scheduler has third position according to its performance in throughput fairness. The RR assigns resources more fairly among UEs but fair resource assignment does not guarantee fair throughput distribution. The B-CQI scheduler came last in terms of throughput fairness.

Table II: Performance Evaluation of Proposed Scheme and conventional schedulers in normal mode

\begin{tabular}{|c|c|c|c|c|}
\hline Scheduler & $\begin{array}{c}\text { Average System } \\
\text { Throughput } \\
\text { (Mbps) }\end{array}$ & $\begin{array}{c}\text { Throughput } \\
\text { Fairness } \\
\text { Index (0-1) }\end{array}$ & $\begin{array}{c}\text { Average } \\
\text { Packet } \\
\text { Delay (ms) }\end{array}$ & $\begin{array}{c}\text { Packet } \\
\text { Loss } \\
\text { Rate }\end{array}$ \\
\hline $\begin{array}{c}\text { Proposed } \\
\text { Scheduler }\end{array}$ & 7.92 & 0.9216 & 1.46 & 0 \\
\hline $\begin{array}{c}\text { Proportional } \\
\text { Scheduler }\end{array}$ & 9.74 & 0.8223 & 1.93 & 0 \\
\hline $\begin{array}{c}\text { Round } \\
\text { Robin }\end{array}$ & 5.44 & 0.7642 & 0.83 & 0 \\
\hline Best CQI & 15.62 & 0.2382 & 18.89 & 0.1427 \\
\hline
\end{tabular}

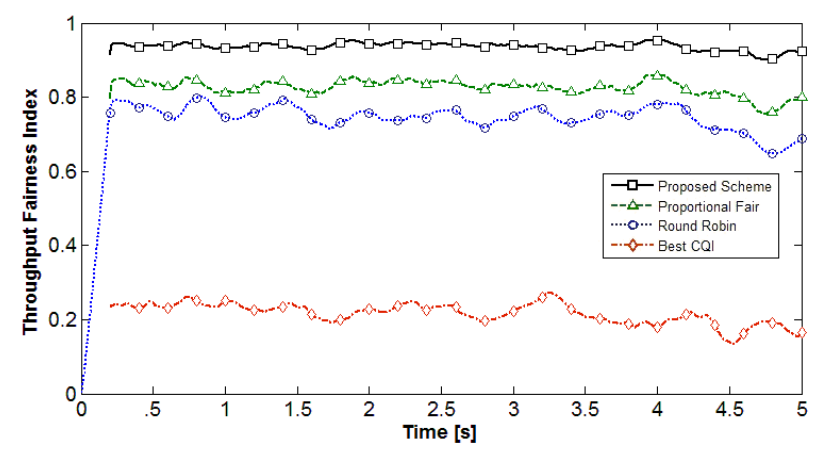

Figure 3: Downlink Throughput Fairness Index with no DRX Sleep.

Table II summarizes the results expressed in Figure 2 and Figure 3 with average values of system throughput and throughput fairness index. The average value of packet delays show that the RR scheduler achieved least delay value followed by Proposed Scheme, which has performed better than PF and B-CQI scheduler. The B-CQI scheduler performed worst in this regard because it ignores UEs with bad channel conditions. It had a packet loss rate of 0.1427 that means $14.27 \%$ of its packets were lost because packet delay had reached more than the delay threshold.

\section{B. Power Saving Mode}

To evaluate the performance of schedulers when considered power saving environment, DRX is implemented on the UE with DRX on-duration set to 1 TTI, active duration to 4 TTI and sleep duration to 7 TTI.

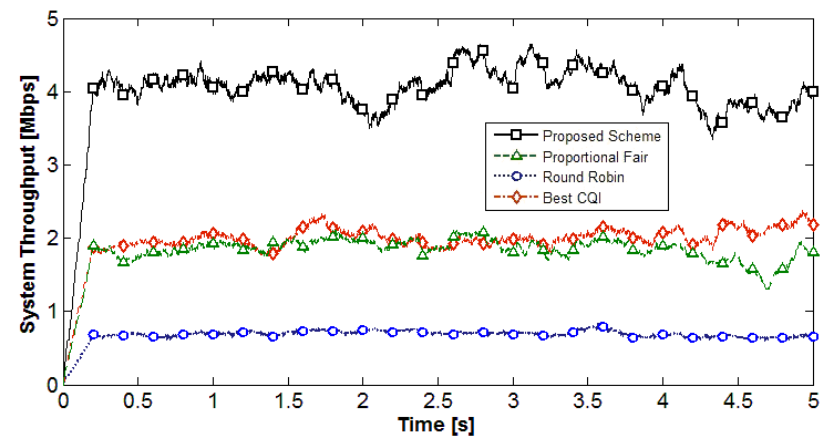

Figure 4: Downlink System Throughput with DRX sleep mode.

Figure 4 shows the throughput of the Proposed Scheme is significantly higher than all other three scheduler because it uses the DRX information for each UE. The UEs who are either in On-duration or In-active duration are considered in the scheduling decision (see $\delta$ in equations (4) and (5)). The other schedulers are not designed in this way; they consider all connected UEs during scheduling. The B-CQI has the highest dropped in system throughput as compare to other schemes. In this simulation, throughput of the PF and $\mathrm{B}-\mathrm{CQI}$ has dropped 
significantly as compared to the case of without the DRX mode. Figure 4 depicts that system throughput of PF and B$\mathrm{CQI}$ is nearest to each other because both schedulers do not consider the delay factor.

Figure 5 shows the graph of Throughput Fairness Index according to Jain's equation. The Proposed Scheme manages to achieve higher fairness because it considers channel conditions and UE's GBR requirements. It tries to allocate resources so that every UE should achieve throughput higher than defined GBR value. The PF scheduler takes second position, and its average fairness index is very close to Proposed Scheme because this scheduler tries to achieve balance between highest achievable throughput and fairness. The RR scheduler is in the third position; theoretically, RR should achieve maximum throughput fairness, but it does not consider channel conditions therefore is staying behind Proposed scheduler. Finally, the value of $\mathrm{B}-\mathrm{CQI}$ scheduler is lowest and is close to the worst case scenario.

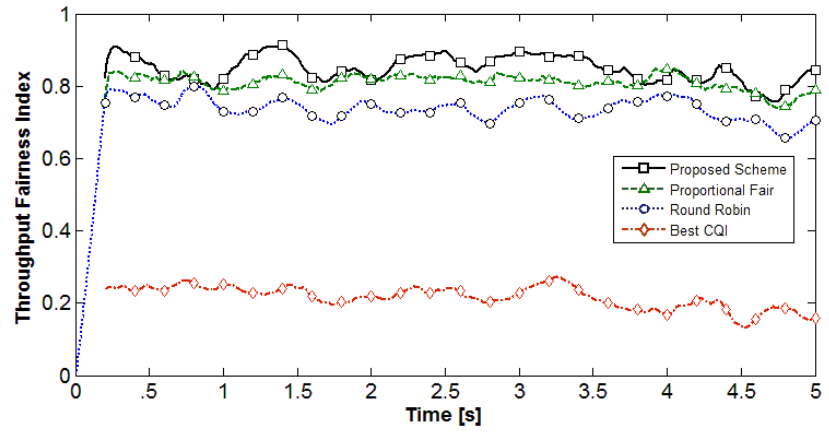

Figure 5: Downlink Throughput Fairness Index with DRX sleep modes.

Table III summarizes the performance characteristics of all the schedulers. The Proposed Scheme, performed not as well as the RR scheduler in terms of packet delay. The RR scheduler achieved the poorest throughput and lower fairness index value because it does not exploit the UEs with good channel conditions, rather it treats them equally. There is no packet loss occurred during the simulation in the first three schedulers as shown in Table III. The B-CQI lost 32\% of its generated packets as it disregards poor channel quality UEs.

Table III: Performance Evaluation of Proposed Scheme and conventional schedulers with DRX Sleep mode

\begin{tabular}{|c|c|c|c|c|}
\hline Scheduler & $\begin{array}{c}\text { Average System } \\
\text { Throughput } \\
\text { (Mbps) }\end{array}$ & $\begin{array}{c}\text { Throughput } \\
\text { Fairness } \\
\text { Index (0-1) }\end{array}$ & $\begin{array}{c}\text { Average } \\
\text { Packet } \\
\text { Delay (ms) }\end{array}$ & $\begin{array}{c}\text { Packet } \\
\text { Loss } \\
\text { Rate }\end{array}$ \\
\hline $\begin{array}{c}\text { Proposed } \\
\text { Scheduler }\end{array}$ & 4.15 & 0.8337 & 5.48 & 0 \\
\hline $\begin{array}{c}\text { Proportional } \\
\text { Scheduler }\end{array}$ & 1.82 & 0.8033 & 6.33 & 0 \\
\hline $\begin{array}{c}\text { Round } \\
\text { Robin }\end{array}$ & 0.67 & 0.7596 & 3.92 & 0 \\
\hline Best CQI & 1.94 & 0.2418 & 21.83 & 0.3268 \\
\hline
\end{tabular}

\section{Power saving and $Q o S$}

The effect of the power saving mechanism on QoS in LTE networks will be evaluated with shorter simulation duration of 1000 TTI. The simulation setup is the same for all the schedulers like the previous simulations. All schedulers will be subjected to different lengths of DRX cycles; the On-duration and active durations are same as previous simulation, only sleep durations are being changed. The DRX Sleep duration will be further increased; longer sleep duration implies more power being saved.

Figure 6 depicts throughput curves for Proposed Scheme and the traditional schedulers. Results are taken by changing the lengths of DRX Sleep duration. Proposed Scheme did not perform best when no DRX (sleep duration $=0$ ) is used. This is due to the fact that Proposed Scheme is designed to provide better fairness among the UEs by fulfilling GBR UEs requirements at the cost of lower system throughput. Here it is clearly seen that the Proposed Scheme is least affected by the increase in sleep durations because it considers DRX state of UEs. The B-CQI and PF scheduler perform better than the Proposed Scheme in normal mode of operation, but their performance degraded significantly when the system is working in power saving mode. Figure 6 clearly shows that the Proposed Scheme is still performing better than other schemes if duration of DRX sleep is increased.

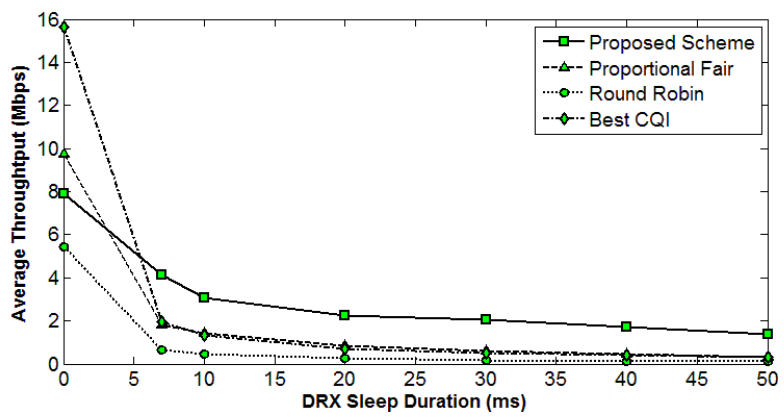

Figure 6: System Throughput vs. Power Saving.

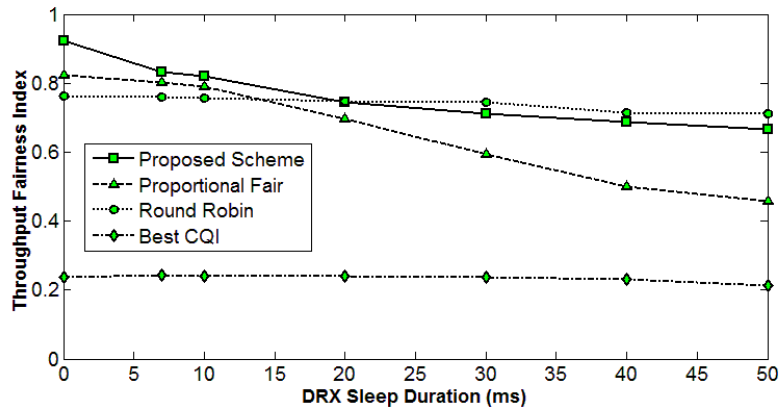

Figure 7: Throughput Fairness Index vs. Power Saving.

Figure 7 shows that initially the Proposed Scheme performs significantly well, but its fairness index reduces and goes below RR after $20 \mathrm{~ms}$ sleep duration. When the sleep 
duration increases, packet delays increase and scheduler tries to compensate this by giving more resources to the UE which packet is getting closer to the delay threshold. As a result, some UEs have higher throughput and some experience lower throughput. Figure 7 clearly shows that the RR has the highest performance index as compared to the Proposed Scheme but the RR did not provide sufficient data rates for UEs to satisfy its QoS requirements, which are shown in Figure 6. The PF performance started to degrade after $10 \mathrm{~ms}$ sleep duration. The $\mathrm{B}-\mathrm{CQI}$ performed the worst in this case due to its resource distribution policy.

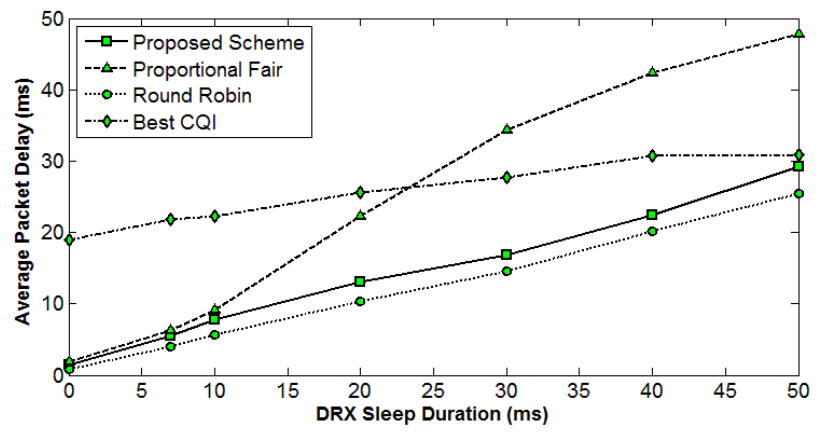

Figure 8: Average Packet Delay vs. Power Saving.

Figure 8 shows the effect of power saving on packet delays for all four schedulers. The packet delay threshold for VoIP is $100 \mathrm{~ms}$ according to LTE QCI otherwise the packet will be discarded. When the DRX sleep duration increases, the most of the time UE switched off which result in packets start to get delayed. Figure 8 shows that RR performed best and Proposed Schemes performed second best. Both of these curves followed a linear pattern while the PF initially started well, but its performance degraded significantly after $20 \mathrm{~ms}$ sleep duration. Similar behavior can be observed in Figure 7 when throughput Fairness index decreased rapidly after $20 \mathrm{~ms}$ sleep duration. The B-CQI performed worst in terms of packet delays because it is designed to achieve maximum systems throughput thus it disregards fairness and delay constraints.

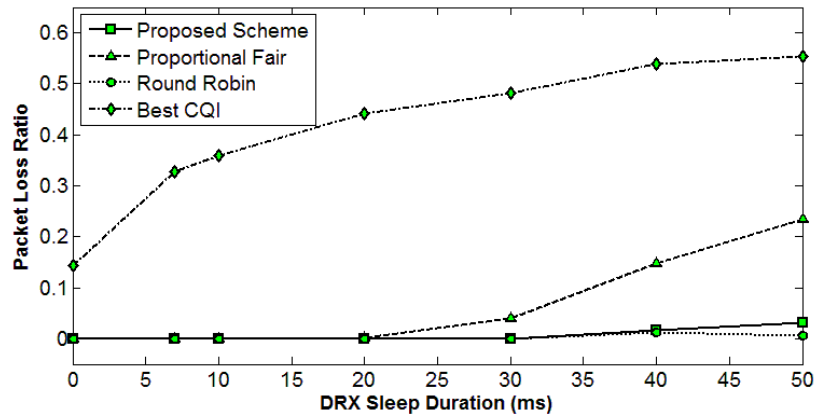

Figure 9: Packet Loss Rate vs. Power Saving.

Figure 9 depicts the performance of the schedulers in terms of packet loss rate. If a packets delay exceeds the delay threshold (100 ms for VoIP) then it is considered to be a lost packet. The RR scheduler achieved zero packet loss until 30 ms sleep duration, after that it started to lose a few packets. The Proposed Scheme performed second best, but it performed very close to the RR scheduler. The Proposed Scheme is designed to reduce packet delays and losses while achieving good throughput and fairness (see delay_factor in Equation (5)). The PF scheduler performed well in low power saving conditions, but its packet loss rate started to increase rapidly after $20 \mathrm{~ms}$ sleep duration. The B-CQI performed worst in terms of packet loss rate because it serves only few UEs which have good channel condition while packets of others UEs get lost due to insufficient resources.

\section{CONCLUSION}

In this paper, we proposed a new QoS-aware downlink scheduling algorithm for delay sensitive traffic (VoIP). The Proposed Scheme endeavors to provide better QoS by decreasing packet losses, improve fairness among the UE and considering the QoS requirement of multimedia service. It has the capability to assure QoS in both power saving and non-power saving environment. The Proposed Scheme is compared with the traditional schemes according to different QoS attributes through simulations. The Proposed Scheme is evaluated in normal operation mode and power saving mode and the impact of power saving on QoS is also examined. In a normal nonpower saving environment, the Proposed Scheme performs well in terms of throughput fairness among the UEs with acceptable packet delay. In a power saving environment, its performance is remarkably better than traditional schedulers because it allocates resources efficiently among the UE.

In future work, a longer simulation environment will be used with multiple eNodeBs. The mobility effect on QoS will be evaluated by considering the handover procedure. The performance of Proposed Scheme will be examined with Deep Sleep mode of operation and its comparison with DRX Light Sleep.

\section{ACKNOWLEDGMENT}

Scott Fowler was partially supported by the Swedish Excellence Center at Linköping - Lund in information Technology (ELLIIT) and EC-FP7 Marie Curie CIG grant, Proposal number: 294182.

\section{REFERENCES}

[1] Kian Chung Beh, S. Armour, and A. Doufexi. Joint time-frequency domain proportional fair scheduler with harq for 3gpp lte systems. In Vehicular Technology Conference, 2008. VTC 2008-Fall. IEEE 68th, pages $1-5$, Sept. 2008.

[2] Huang Bo, Tian Hui, Chen Lan, and Zhu Jianchi. Drx-aware scheduling method for delay-sensitive traffic. Communications Letters, IEEE, 14(12):1113 - 1115, December 2010.

[3] Yong Fan, P. Lunden, M. Kuusela, and M. Valkama. Efficient semipersistent scheduling for voip on eutra downlink. In Vehicular Technology Conference, 2008. VTC 2008-Fall. IEEE 68th, pages 1 - 5, Sept. 2008.

[4] S. Fowler. Study on power saving based on radio frame in lte wireless communication system using drx. IEEE GLOBECOM Workshops (GC Wkshps), December 2011.

[5] Scott Fowler, Ranjeet S Bhamber, and Abdelhamid Mellouk. Analysis of adjustable and fixed drx mechanism for power saving in lte/lte-advanced. IEEE International Conference on Communications (ICC), June 2012. 
[6] R. Jain. The art of computer systems performance analysis: techniques for experimental design, measurement, simulation and modeling. New York, John Wiley \& Sons, 1991.

[7] Yan Lin and Guangxin Yue. Channel-adapted and buffer-aware packet scheduling in lte wireless communication system. In Wireless Communications, Networking and Mobile Computing, 2008. WiCOM '08. 4th International Conference on, pages 1 - 4, Oct. 2008.

[8] K.I. Pedersen, T.E. Kolding, F. Frederiksen, I.Z. Kovacs, D. Laselva, and P.E. Mogensen. An overview of downlink radio resource management for utran long-term evolution. Communications Magazine, IEEE 47(7):86 - 93, July 2009.

[9] H.A.M. Ramli, R. Basukala, K. Sandrasegaran, and R. Patachaianand. Performance of well known packet scheduling algorithms in the downlink 3gpp lte system. In Communications (MICC), 2009 IEEE 9th Malaysia International Conference on, pages 815 - 820, dec. 2009.

[10] J. Wigard, T. Kolding, L. Dalsgaard, and C. Coletti. On the user performance of lte ue power savings schemes with discontinuous reception in 1te. In Communications Workshops, 2009. ICC Workshops 2009. IEEE International Conference on, pages 1 - 5, June 2009. 